\begin{tabular}{|c|l|}
\hline Title & $\begin{array}{l}\text { Probing the Molecular Conformation of Self-A ssembled Monolayers at Metal Semiconductor Interfaces by Vibrational } \\
\text { Sum Frequency Generation Spectroscopy }\end{array}$ \\
\hline Author(s) & A sanuma, Hidehiko; Noguchi, Hidenori; Huang, Y ifan; Uosaki, Kohei; Yu, HuzZ Zhong \\
\hline Citation & $\begin{array}{l}\text { Journal of Physical Chemistry C, 113(50), 21139-21146 } \\
\text { https:/doi.org/10.1021/jp9062675 }\end{array}$ \\
\hline Issue Date & 2009-12-17 \\
\hline Doc URL & http://hdl.handle.net/2115/50209 \\
\hline Type & article \\
\hline File Information & JPCC113-50_21139-21146.pdf \\
\hline
\end{tabular}

Instructions for use 


\title{
Probing the Molecular Conformation of Self-Assembled Monolayers at Metal/ Semiconductor Interfaces by Vibrational Sum Frequency Generation Spectroscopy
}

\author{
Hidehiko Asanuma ${ }^{\dagger}$ Hidenori Noguchi, ${ }^{\dagger}$ Yifan Huang, ${ }^{\dagger}$ Kohei Uosaki, ${ }^{*}$, and Hua-Zhong Yu ${ }^{*, \dagger}$ \\ Department of Chemistry, Simon Fraser University, Burnaby, British Columbia V5A 1S6, Canada, and Division \\ of Chemistry, Graduate School of Science, Hokkaido University, Sapporo 060-0180, Japan
}

Received: July 3, 2009; Revised Manuscript Received: October 15, 2009

\begin{abstract}
The conformation of self-assembled monolayers (SAMs) buried at metal-semiconductor interfaces plays a crucial role in the functioning of the molecular junctions thus formed. We have studied by vibrational sum frequency generation (SFG) spectroscopy the influence of deposited gold films on the molecular orientation of a series of $n$-alkyl monolayers that are covalently bonded to silicon(111) via $\mathrm{Si}-\mathrm{C}$ linkages $\left(\equiv \mathrm{Si}-\mathrm{C}_{n} \mathrm{H}_{2 n+1}\right.$, $n=10,12,14$, and 18). The SFG spectra indicate that the gold contacts (overlayers) introduce significant gauche defects within the initially highly oriented alkyl chains. The methyl tilt angles of the buried $n$-alkyl monolayers depend on the gold deposition method (vacuum evaporation vs sputtering) and the alkyl chain length. These observations are supported by solid-state electrical measurements.
\end{abstract}

\section{Introduction}

Sum frequency generation (SFG) spectroscopy is based on a second-order nonlinear optical effect in which photons are generated at a frequency equal to the sum of the frequencies of the two incident beams. ${ }^{1-4}$ It is an interface-sensitive technique because the effect is forbidden in media exhibiting inversion symmetry; at a surface or interface such inversion symmetry is necessarily broken. By using visible and tunable infrared input beams, SFG can detect specific vibrations at a buried interface at the time resolution of the laser source. In addition, the polarization combinations of SFG, visible and IR beams can be powerful diagnostic tools for the determination of molecular orientations: the $s s p$ (s-polarized SFG, $s$-polarized visible, and $p$-polarized IR) combination detects vibrational modes with dipole moments perpendicular to the interface, the sps and pss combinations are sensitive to dipole moments parallel to the surface, and the $p p p$ combination responds to both perpendicular and parallel components. The main advantage of SFG spectroscopy, compared with the conventional infrared techniques (FT-IR and Raman), is its capability to reveal the conformation, lateral order, and orientation of surface-bound molecules.${ }^{5,6}$ For example, Shen and co-workers have determined that the molecular orientations of pentadecanoic acids at a water surface depend on their surface densities; the monolayer undergoes a transition from the highly disordered "liquid expanded" to the "liquid condensed" phase, for which the alkyl chains are extended toward the air and oriented nearly normal to the water surface. ${ }^{1}$

SFG spectroscopy has also been adapted to the study of molecular conformations of self-assembled monolayers (SAMs) formed on various substrates. ${ }^{7-16}$ Two decades ago, GuyotSinnest et al. reported that octadecyltrichlorosilane (OTS) monolayers on silica are oriented normal to the surface ${ }^{8}$ Hines et al. have shown that alkanethiolate SAMs on gold and silver are relatively more ordered than those prepared on platinum;

* To whom correspondence should be addressed. E-mail: hogan_yu@sfu.ca (H.-Z.Y.), uosaki@pcl.sci.hokudai.ac.jp (K.U.).

Simon Fraser University.

${ }^{\ddagger}$ Hokkaido University. however, the latter can be "straightened" by applying electrochemical potentials. ${ }^{9}$ Yeganeh et al. discovered that SFG spectra of alkanethiolate monolayers on a gold(111) surface exhibit an azimuthal angle dependence and attributed this behavior to the binding pattern of the molecules. ${ }^{16} \mathrm{Liu}$ et al. studied the role of water during the adsorption of OTS on a fused silica surface by monitoring conformational changes of the monolayers. ${ }^{11}$ Recently, Ye et al. examined the orientation of water molecules at the surface of OTS monolayers on quartz under neutral and acidic conditions. ${ }^{14,15}$ Nishi et al. found that the ratio between $\mathrm{CH}_{3}$ symmetric and asymmetric vibration modes of alkanethiolate SAMs on gold depends on whether the number of carbons is odd or even; this may be due to the difference in the tilt direction of the terminal methyl groups. ${ }^{13}$

The miniaturization of electronic devices in the past decade has increased the demand for fabricating nanoscale structures, in which organic molecules act as either passive (i.e., tunneling junctions, rectifiers) or active components (i.e., switches, transistors, and logic gates). ${ }^{17}$ Organic molecules are generally assembled into monolayers whose orientation and conformation play a crucial role in the functioning of the molecular junctions thus formed. Several spectroscopic studies of SAMs at metal/ metal or metal/semiconductor interfaces have been reported recently. ${ }^{18-23}$ While Jun and Zhu developed an attenuated total reflection (ATR) method to probe buried molecular interfaces by IR spectroscopy ${ }^{20} p$-polarized backside reflection absorption infrared spectroscopy (pb-RAIRS) has been adapted by Richter et al. to the study of the interactions between deposited metal contacts and organic monolayers on silicon surfaces. ${ }^{21}$ These investigations typically rely on observed peak broadening, frequency shifts, and intensity variations. Hacker et al. have reported the complete disappearance of methylene bands from the IR spectrum of an octadecyl monolayer on silicon upon the deposition of a $5 \mathrm{~nm}$ gold film. ${ }^{22}$

In this work, we explore vibrational SFG spectroscopy as a new technique to probe the molecular orientation of selfassembled monolayers at metal/semiconductor interfaces. The samples were prepared by depositing gold contacts onto alkyl monolayers covalently bonded to silicon surfaces. ${ }^{23,24} \mathrm{We}$ examined not only the effect of the alkyl chain length but also 
the influence of the coating method (thermal evaporation vs sputtering deposition) on the electrical properties of the $\mathrm{Au} /$ alkyl monolayer/Si junctions formed. Besides recording changes of various vibrational band frequencies and intensities due to the $\mathrm{CH}_{3}$ and $\mathrm{CH}_{2}$ groups, we were able to monitor the $\mathrm{Au}-\mathrm{CH}$ interactions directly by combining SFG with ATR-IR spectroscopic studies.

\section{Experimental Section}

2.1. Materials. All chemicals were ACS reagent grade and used as received unless otherwise mentioned. Deionized water $(>18.3 \Omega \cdot \mathrm{cm})$ was used throughout the experiments. 1-Decene (94\%), 1-dodecene (95\%), 1-tetradecene (92\%), 1-octadecene (90\%), and 1,1,1,-trichloroethane $(99.5 \%)$ were obtained from Aldrich; tetrahydrofuran (THF), sulfuric acid (96\%), and hydrogen peroxide $(30 \%)$ were purchased from Wako Chemicals. Ammonium fluoride (40\%) was from Morita Chemical Industries. 1-Decene (94\%), 1-dodecene (95\%), 1-tetradecene $(92 \%)$, and 1-octadecene $(90 \%)$ were distilled from sodium under reduced pressure (20-30 Torr), followed by freeze-thaw cycles under vacuum/nitrogen.

2.2. Surface Preparation. Silicon(111) wafers (0.5-5.0 $\Omega \cdot \mathrm{cm}$, n-type, Virginia Semiconductor Inc.) were cut to appropriate size $(1.5 \times 2 \mathrm{~cm})$ and then cleaned in a "piranha" solution (3:1 mixture of $\mathrm{H}_{2} \mathrm{SO}_{4}$ and $\mathrm{H}_{2} \mathrm{O}_{2}$ ) for 30 min at $90{ }^{\circ} \mathrm{C}$. CAUTION: "Piranha" solution must be handled with extreme care as it reacts violently with organic materials. After copious rinsing with deionized water, the silicon wafers were etched with deoxygenated $\mathrm{NH}_{4} \mathrm{~F}$ ( $40 \%$ aqueous solution) to remove native oxide and obtain hydrogen-terminated silicon $(\equiv \mathrm{Si}-\mathrm{H})$. The fresh $\equiv \mathrm{Si}-\mathrm{H}$ samples were then introduced under argon into Schlenk tubes containing $2-3 \mathrm{~mL}$ of deoxygenated neat 1-alkenes and heated to $160{ }^{\circ} \mathrm{C}$ for $4 \mathrm{~h}$. Thus, modified silicon samples ( $\equiv \mathrm{Si}-\mathrm{C}_{n} \mathrm{H}_{2 n+1}, n=10,12,14$, and 18 ; abbreviated as $\mathrm{C} 10, \mathrm{C} 12, \mathrm{C} 14$, and $\mathrm{C} 18$, respectively) were cleaned with THF, trifluororoacetic acid solution in THF (1\%), and 1,1,1-trichloroethane sequentially and blow-dried with $\mathrm{N}_{2}$ gas.

Gold deposition on freshly prepared silicon samples was performed via thermal evaporation or sputtering coating. Thermal evaporation was carried out in a vacuum evaporation apparatus (Ulvac, EBH-6). The chamber pressure was lowered to the base pressure of $10^{-6}$ Torr using a diffusion pump; gold $(99.99 \%)$ was then deposited at a rate of $0.1 \mathrm{~nm} / \mathrm{s}$ until it reached the film thickness of $10 \mathrm{~nm}$ (measured by a calibrated quartz crystal oscillator). Gold sputtering was carried out with a Joel JFC-1200 sputtering coater in an argon-purged chamber under reduced pressure $\left(\sim 2.0 \times 10^{-2}\right.$ Torr $)$. The filament current was kept at approximately $15 \mathrm{~mA}$ until the desired film thickness $(\sim 10 \mathrm{~nm})$ was reached. The size of gold contacts was controlled by using an aluminum mask with nine holes ( $3 \mathrm{~mm}$ diameter).

2.3. SFG System. A picosecond Nd:YAG laser (PL2143B, Ekspla) was used to pump an optical parametric generation/ optical parametric amplification/difference frequency generation (OPG/OPA/DFG) system to produce tunable infrared radiation $(2.3-8.5 \mu \mathrm{m}) .{ }^{14,15,25}$ The second harmonic output of the YAG (yttrium-aluminum-garnet) laser at $532 \mathrm{~nm}$ was used as the visible light source. At the incident angle of $70^{\circ}$ for visible and of $50^{\circ}$ for infrared light, two beams were loosely focused onto the sample. The [211] direction of $\mathrm{Si}(111)$ samples was set in the plane of the incident beams during measurements. The produced SFG signal was filtered through irises and a monochromator (Oriel Instruments, MS257) and amplified by a photomultiplier tube (PMT, Hamamatsu, R3896). All measurements were carried out with $p$-polarized SFG, visible and IR beams (abbreviated $p p p$ ). The SFG signal was normalized to the intensities of the visible and infrared light; all experiments were carried out in air at room temperature $\left(22 \pm 2{ }^{\circ} \mathrm{C}\right)$.

The following formulas were used to fit the SFG intensity $\left(I_{\mathrm{SFG}}\right)$

$$
I_{\mathrm{SFG}}\left(\omega_{\mathrm{SFG}}=\omega_{\mathrm{IR}}+\omega_{\mathrm{VIS}}\right) \propto\left|\chi_{\mathrm{eff}}^{(2)}: E\left(\omega_{\mathrm{IR}}\right) E\left(\omega_{\mathrm{VIS}}\right)\right|^{2}
$$

$$
\chi_{\mathrm{eff}}^{(2)}=\left|\chi_{\mathrm{NR}}^{(2)}\right| e^{i \phi_{n}}+\sum \frac{A_{n}}{\omega_{\mathrm{IR}}-\omega_{n}+i \Gamma_{n}}
$$

where $\chi_{\mathrm{eff}}^{(2)}$ and $\chi_{\mathrm{NR}}^{(2)}$ are the effective second-order nonlinear susceptibilities of the resonant and nonresonant component, respectively, $\phi_{n}$ is the phase angle between the resonant and nonresonant component, and $A_{n}$ and $\Gamma_{n}$ are the amplitude and the homogeneous width of the surface vibration mode $(n)$ corresponding to the frequency $\omega_{n}$, respectively. On the basis of the above equations, a nonlinear least-squares routine was developed in our laboratory to fit the SFG spcetra. ${ }^{14,15}$ The quality of the fit is judged by $r^{2}$ values that were at least 0.900 . Specifically, for each vibrational band, the fitting parameters were $A_{n}, \omega_{\mathrm{IR}}$, and $\Gamma_{n}$, while the $\chi_{\mathrm{NR}}^{(2)}$ and $\phi_{n}$ were kept constant for the entire spectrum. Another parameter (a constant for flat and a linear term for uneven baselines) was added to adjust the baseline, and this term does not influence the spectral features. Peak intensity ratios were calculated from the amplitudes of individual vibration modes $\left(A_{n}\right)$. All uncertainties were derived from the spectra of at least three samples.

2.4. Electrical Measurements. The solid-state electrical measurements were carried out with an Autolab electrochemical analyzer (model PGSTAT 30, Eco Chemie BV, The Netherlands). All measurements were performed in a Faraday cage. The contact to the gold pad was made by lowering a tungsten probe with a homemade micropositioning system. The currentvoltage $(I-V)$ curves were recorded from +0.5 to $-0.5 \mathrm{~V}$ at a scan rate of $0.05 \mathrm{~V} / \mathrm{s}$. The Mott-Schottky plots $\left(C^{-2}\right.$ vs $\left.V\right)$ were acquired by scanning the potential from +0.1 to $-1.5 \mathrm{~V}$ at a frequency of $100 \mathrm{kHz}$.

2.5. Other Measurements. Wetting measurements were carried out with an AST Optima contact angle system at ambient conditions $\left(18-23{ }^{\circ} \mathrm{C}, 30-35 \%\right.$ relative humidity) using a horizontal light beam to illuminate a $2.0 \mu \mathrm{L}$ water droplet. Ellipsometric measurements were performed with a Uvisel spectroscopic ellipsometer (Horiba Jobin Yvon) over the range of 300-700 nm at an incident angle of $70^{\circ}$. All reported experimental uncertainties are derived from at least three spots from three independent samples.

\section{Results and Discussion}

Previous FT-IR spectroscopic studies of self-assembled monolayers (SAMs) at metal/metal (semiconductor) interfaces have provided valuable information regarding metal-molecule interactions, based on the attenuation of certain vibrational modes, peak broadening, or position shifts. For example, Boer et al. proposed that the decrease of the IR peak intensity of the $-\mathrm{CH}_{3}$ groups of 1-hexadecanethiolate monolayers on gold is due to the $\mathrm{Au} / \mathrm{Al}$ and $\mathrm{CH}_{3}$ interactions; ${ }^{18}$ Jun and Zhu have suggested that the peak broadening observed for metal/OTS/ silicon interfaces is due to monolayer disordering and the peak attenuation due to the cleavage of $\mathrm{C}-\mathrm{H}$ bonds. ${ }^{20}$ These observations may not be simply explained because the dipoles of molecules can be "suppressed" by the metal overlayers, and the roughness of the metal layer may induce scattering of the infrared beam at the metal/molecule interface. ${ }^{26}$ There are cases 


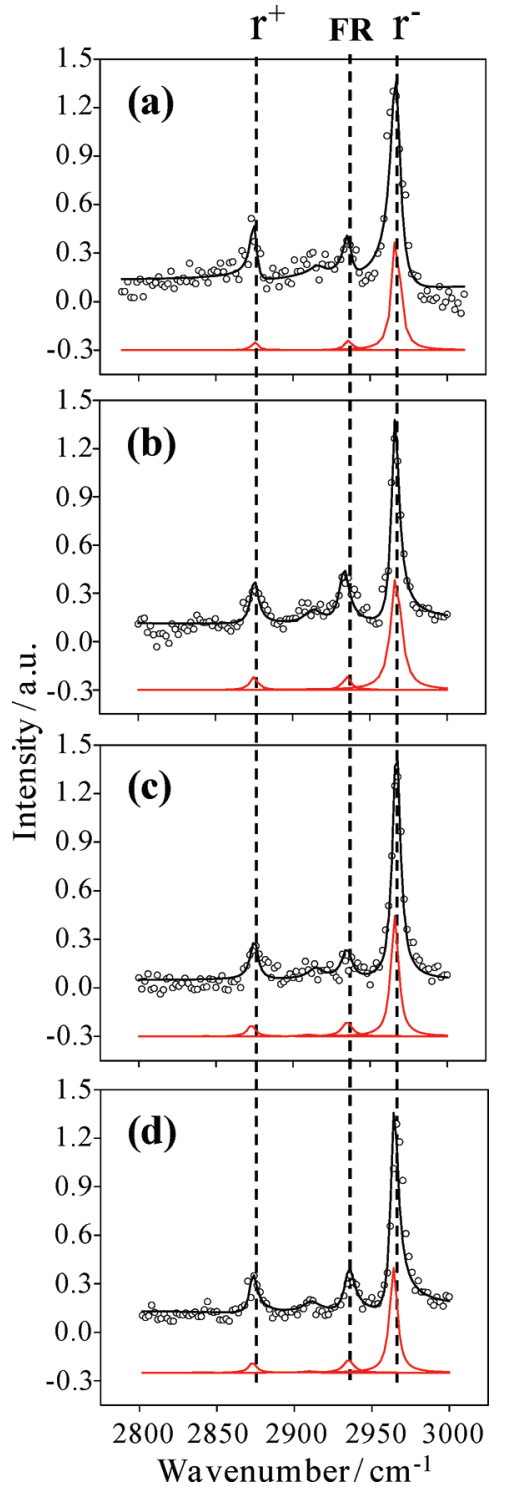

Figure 1. SFG spectra of (a) $\equiv \mathrm{Si}-\left(\mathrm{CH}_{2}\right)_{9} \mathrm{CH}_{3} \quad$ (C10), (b) $\equiv \mathrm{Si}-\left(\mathrm{CH}_{2}\right)_{11} \mathrm{CH}_{3} \quad(\mathrm{C} 12), \quad(\mathrm{c}) \equiv \mathrm{Si}-\left(\mathrm{CH}_{2}\right)_{13} \mathrm{CH}_{3} \quad(\mathrm{C} 14)$, and (d) $\equiv \mathrm{Si}-\left(\mathrm{CH}_{2}\right)_{17} \mathrm{CH}_{3}(\mathrm{C} 18)$. The solid lines (in black) show the best fits to the experimental data (open circles) based on eqs 1 and 2; the deconvoluted peaks are shown in red at the bottom (see the main text for details).

where new bands arise upon the deposition of metal atoms on top of the organic monolayer; Richter et al. assigned the new peak at $\sim 2825 \mathrm{~cm}^{-1}$ from the metal/OTS/Si system to methylene-metal interactions. ${ }^{21}$ Nevertheless, the abovementioned spectral features are not easily distinguishable as they are typically broad, weak, and sometimes obscured. SFG spectroscopy can provide additional or complementary information to these IR studies, as it is intrinsically sensitive to the interfacial molecular conformation and exhibits submonolayer sensitivity. ${ }^{27}$

3.1. Alkyl Monolayers on Silicon. The $\mathrm{Si}-\mathrm{C}$-bonded alkyl monolayers were first examined by SFG spectroscopy prior to the metal deposition (Figure 1). All spectra exhibited features that correspond to densely packed and well-oriented monolayers. . $^{10,12,25}$ Specifically, contributions from methylene $\left(\mathrm{CH}_{2}\right)$ stretching modes were negligible, and the spectral features were dominated by the contributions from the methyl $\left(\mathrm{CH}_{3}\right)$ groups: methyl symmetric stretch $\left(\mathrm{r}^{+}\right)$at $\sim 2878 \mathrm{~cm}^{-1}$, Fermi resonance (FR)
TABLE 1: Thickness and Wetting Measurements of Alkyl Monolayers on Silicon

\begin{tabular}{lccc}
\hline sample & $\mathrm{H}_{2} \mathrm{O}$ contact angle/degree & $d_{\text {exp }} / \AA$ & $d_{\text {cald }} / \AA^{a}$ \\
\hline$\equiv \mathrm{Si}-\left(\mathrm{CH}_{2}\right)_{9} \mathrm{CH}_{3}(\mathrm{C} 10)$ & $109 \pm 2$ & $12.8 \pm 1.0$ & 11.4 \\
$\equiv \mathrm{Si}-\left(\mathrm{CH}_{2}\right)_{11} \mathrm{CH}_{3}(\mathrm{C} 12)$ & $108 \pm 3$ & $14.0 \pm 1.2$ & 13.5 \\
$\equiv \mathrm{Si}-\left(\mathrm{CH}_{2}\right)_{13} \mathrm{CH}_{3}(\mathrm{C} 14)$ & $109 \pm 2$ & $15.4 \pm 1.1$ & 15.7 \\
$\equiv \mathrm{Si}-\left(\mathrm{CH}_{2}\right)_{17} \mathrm{CH}_{3}(\mathrm{C} 18)$ & $106 \pm 2$ & $20.4 \pm 1.3$ & 20.0
\end{tabular}

${ }^{a}$ The predicted monolayer thickness was calculated from the equation $d=1.86+1.30(n-1) \cos \theta$, where $d$ is the thickness, $n$ is the number of carbons in the alkyl chain, and $\theta$ is the angle between the molecule axis and the surface normal. An average tilt angle, $\theta=35^{\circ}$, was used to calculate the thickness of each alkyl monolayer.

between $\mathrm{r}^{+}$and the $\mathrm{CH}$ bending overtone at $\sim 2940 \mathrm{~cm}^{-1}$, and the $\mathrm{CH}_{3}$ asymmetric stretch $\left(\mathrm{r}^{-}\right)$at $\sim 2964 \mathrm{~cm}^{-1}$. These are the features of well-ordered and closely packed molecular monolayers that have the alkyl chains in all-trans configurations. The ellipsometric thickness and the wetting measurements summarized in Table 1 support the above interpretation. The monotonic thickness increase of the $\mathrm{C} 10$ to $\mathrm{C} 18$ monolayers with the alkyl chain length is in good agreement with values calculated for an average alkyl chain tilt angle of $35^{\circ}$ (corresponding to a $\mathrm{CH}_{3}$ tilt angle of $\sim 70^{\circ}$ ) (Table 1). The static water contact angles were around $110^{\circ}$ for all four monolayer systems, comparable to previous reports for well-packed (intrinsically hydrophobic) alkyl monolayers. ${ }^{24,28-30}$ ATR - IR studies provide additional evidence (e.g., strong $\mathrm{CH}_{2}$ asymmetric and symmetric stretching bands) for the high quality of these monolayers (see the Supporting Information).

3.2. Alkyl Monolayers at Gold/Silicon Interfaces. Figures 2 and 3 show the SFG spectra of the C10, C12, C14, and C18 monolayers on silicon with deposited gold overlayers. The samples were prepared by thermal evaporation and sputtering deposition. In contrast to Figure 1, the vibrational bands appear as downward peaks due to a phase difference between the nonresonant background (contributed largely from the gold overlayer) and the resonant signal, ${ }^{31,32}$ which is also related to the laser configuration ${ }^{9}$ and has been observed for SFG spectra of alkanethiol SAMs on gold. ${ }^{9,32}$ In general, upon gold deposition, strong $\mathrm{CH}_{2}$ symmetric $\left(\mathrm{d}^{+}\right)$and asymmetric $\left(\mathrm{d}^{-}\right)$peaks appear at $\sim 2850$ and $\sim 2920 \mathrm{~cm}^{-1}$. The methylene groups of well-ordered alkyl chains are positioned in a symmetric manner, yet when gauche defects are introduced, such symmetry is broken and the SFG signal arises. Therefore, these new bands indicate that trans-gauche defects in the alkyl chain backbones are introduced upon metal deposition. ${ }^{25,33}$ Independent of the alkyl chain lengths, both thermal evaporation and sputtering deposition of gold contacts disrupt the molecular conformation of the SAMs significantly, as evidenced by the substantial contributions of the methylene bands $\left(\mathrm{d}^{+}\right.$and $\left.\mathrm{d}^{-}\right)$. As shown by the deconvoluted peaks (Figures 2 and 3), methylene contributions relative to methyl peaks are observed to be stronger for samples prepared via sputtering than for those prepared by thermal evaporation; that is, the sputtering method is more destructive. Another important feature of these SFG spectra is the appearance of a new band at $\sim 2900 \mathrm{~cm}^{-1}$. Although $\mathrm{d}^{+}$ Fermi resonance and several other factors prevent a definite assignment of this band, we have cautiously assigned it to methylene $\left(\mathrm{CH}_{2}\right)$ groups that are interacting with gold, specifically to the $\mathrm{CH}$ bonds that are in the proximity of, but not in direct contact with, gold atoms. ${ }^{34,35}$ This means that, upon metal deposition, some regions of the alkyl chains are not directly in contact with gold. It has been suggested that gold will 


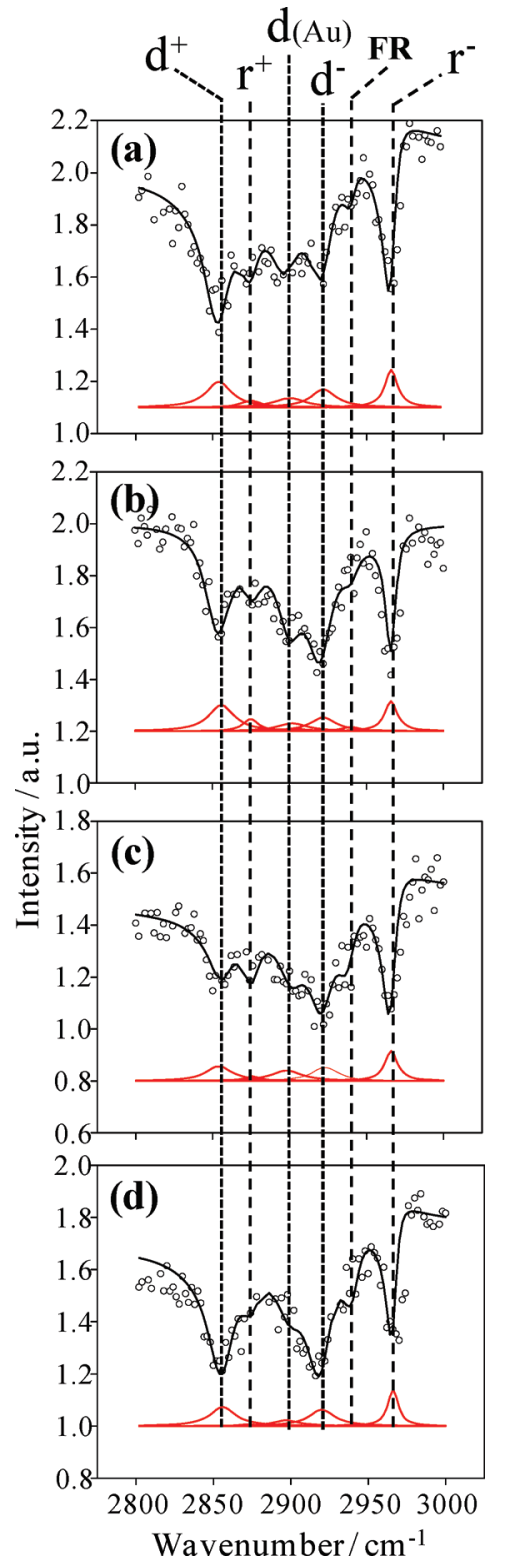

Figure 2. SFG spectra of (a) $\equiv \mathrm{Si}-\left(\mathrm{CH}_{2}\right)_{9} \mathrm{CH}_{3} \quad$ (C10), (b) $\equiv \mathrm{Si}-\left(\mathrm{CH}_{2}\right)_{11} \mathrm{CH}_{3} \quad(\mathrm{C} 12), \quad$ (c) $\equiv \mathrm{Si}-\left(\mathrm{CH}_{2}\right)_{13} \mathrm{CH}_{3} \quad(\mathrm{C} 14)$, and (d) $\equiv \mathrm{Si}-\left(\mathrm{CH}_{2}\right)_{17} \mathrm{CH}_{3}(\mathrm{C} 18)$ upon thermal evaporation of gold overlayers (10 nm thick). The solid lines (in black) show the best fits to the experimental data (open circles) based on eqs 1 and 2; the deconvoluted peaks are shown in red at the bottom (see main text for details).

completely penetrate $n$-alkyl monolayers, ${ }^{22}$ our SFG results show that, at least at the submonolayer scale, gold penetration does occur in a "patchy" manner.

Techniques such as FT-IR spectroscopy and time-of-flight secondary ion mass spectrometry (TOF-SIMS) have helped to detect molecules and determine their chemical identities at buried interfaces. ${ }^{18-21,36}$ The present SFG investigations also provide further insights into molecular orientations. It is clear that the originally highly oriented alkyl chains have become disordered, but the observed strong methyl stretching bands indicate that the organic monolayers are still relatively ordered after the formation of metal contacts to the top.

This is seemingly different from the case reported by Haick et al. in which the metal evaporation damaged the molecules and the decomposition products did not remain at the interface. ${ }^{37}$ Hacker et al. also described the displacement of an entire

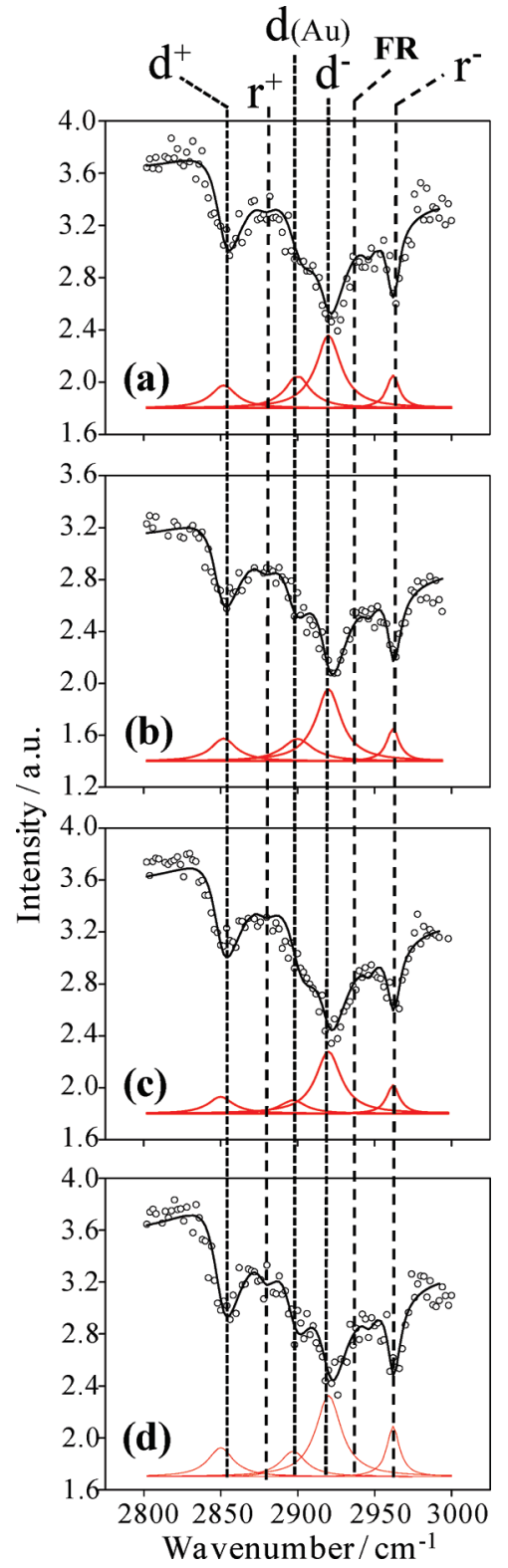

Figure 3. SFG spectra of (a) $\equiv \mathrm{Si}-\left(\mathrm{CH}_{2}\right)_{9} \mathrm{CH}_{3} \quad$ (C10), (b) $\equiv \mathrm{Si}-\left(\mathrm{CH}_{2}\right)_{11} \mathrm{CH}_{3} \quad(\mathrm{C} 12), \quad(\mathrm{c}) \equiv \mathrm{Si}-\left(\mathrm{CH}_{2}\right)_{13} \mathrm{CH}_{3} \quad(\mathrm{C} 14)$, and (d) $\equiv \mathrm{Si}-\left(\mathrm{CH}_{2}\right)_{17} \mathrm{CH}_{3}(\mathrm{C} 18)$ upon sputtering deposition of $10 \mathrm{~nm}$ thick gold overlayers. The solid lines (in black) show the best fits to the experimental data (open circles) based on eqs 1 and 2; the deconvoluted peaks are shown in red at the bottom (see the main text for details).

monolayer detected by $p$-polarized backside reflection absorption infrared spectroscopy (pb-RAIRS). ${ }^{22}$ It should be noted that Haick et al. studied monolayers on GaAs, and Hacker et al. prepared their $\mathrm{Si}-\mathrm{C}$-bonded 1-octadecyl (C18) monolayer with a $10 \mathrm{mM}$ solution of 1-octadecene. ${ }^{22}$ This may account for the observed differences. Our STM studies ${ }^{48}$ and ATR-IR spectroscopic measurements also showed significant differences in the morphology of the gold contacts and in the packing/molcular orientation. Although it is difficult at this stage to conclusively determine the cause of the discrepancies, our results generally support the conclusion drawn by Hacker et al. ${ }^{22}$ that the deposited gold atoms penetrate the monolayer and directly contact the underlying silicon.

3.3. Effect of Metal Deposition Method. To provide further insights into how different metal coating methods influence the molecular conformation of SAMs at buried interfaces, the 
intensity ratio of the methyl symmetric and asymmetric stretches $A_{q}\left(\mathrm{r}^{-}\right) / A_{q}\left(\mathrm{r}^{+}\right)$was used to estimate the average tilt angle using the following equation: ${ }^{38-40}$

$$
\begin{aligned}
& \left|\frac{A_{q}\left(r^{-}\right)}{A_{q}\left(r^{+}\right)}\right|= \\
& \quad\left|\frac{\beta_{c a a}}{\beta_{a a c}} \times \frac{\left(2 F_{z z z}-F_{x x z}\right)\left(\cos \theta-\cos ^{3} \theta\right)}{\left(F_{z z z}+\frac{1}{2} F_{x x z}(1+r)\right) \cos \theta-F_{z z z}-F_{x x z}(1-r) \cos ^{3} \theta}\right|
\end{aligned}
$$

In eq $3, \theta$ is the angle between the main axis of the methyl group and the surface normal; $F_{z z z}$ and $F_{x x z}$ are combinations of the Fresnel factors for the SFG, visible, and IR beams; $\beta$ is the hyperpolarizability; and $r$ is the $\beta_{c c c} / \beta_{a a c}$ ratio (equal to 0.25 ). The ratio $\beta_{c a a} / \beta_{a a c}$ has a value of $4.21 .^{41}$ This model can be applied to the $C_{3 v}$ symmetry for the methyl groups under the assumption that the $z z z$ and $x x z$ susceptibility elements dominate the SFG signal (for gold/silicon interfaces). ${ }^{42}$

$$
F_{z z z}, F_{x x z}>>F_{x z x}, F_{z x x}
$$

Therefore, the tilt angle determination using eq 3 is not applicable to the silicon substrate; it is only valid after deposition of gold that significantly enhances the electric field of the $z$ component. Rather than a particular or an averaged tilt angle, it is more realistic to estimate the range for each monolayer system. Generally, a Gaussian distribution can be assumed to model the methyl orientation angles (see the Supporting Information). ${ }^{43-45}$

$$
\left.f(\theta)=C \exp \left[-\left(\theta-\theta_{0}\right) / 2 \sigma^{2}\right)\right]
$$

In eq $5, \theta_{0}$ is the mean orientation angle, $C$ is a normalization constant, and $\sigma$ is the root-mean-square width. For a direct comparison, thus obtained methyl tilt angles for alkyl monolayers at gold/silicon interfaces are summarized in Table 2.

The average tilt angles of the alkyl chains in alkyl monolayers on silicon have been reported to be $35-40^{\circ},{ }^{12,24,42}$ which corresponds to methyl tilt angles of $70-75^{\circ}$. This means that the alkyl chains become less tilted; that is, they "stand up" from the surface upon metal deposition (Table 2). The absolute tilt angles are difficult to determine due to gauche defects and the wide range of possible orientations. Focusing on the differences between the two gold deposition methods, as shown in Table 2 , the methyl tilt angles for the samples prepared via thermal evaporation are much smaller than those prepared by sputtering
TABLE 2: Estimated Methyl Tilt Angles ( $\theta$ ) of Alkyl Monolayers at Gold/Silicon Interfaces; the Range Was Based on the Assumption of Gaussian Distributions (See Text for Details)

\begin{tabular}{lccccc}
\hline & \multicolumn{2}{c}{ thermal evaporation } & & \multicolumn{2}{c}{ sputtering } \\
\cline { 2 - 3 } \cline { 5 - 6 } sample & $A\left(\mathrm{r}^{-}\right) / A\left(\mathrm{r}^{+}\right)$ & tilt angles & & $A\left(\mathrm{r}^{-}\right) / A\left(\mathrm{r}^{+}\right)$ & tilt angles \\
\hline$\equiv \mathrm{Si}-\left(\mathrm{CH}_{2}\right)_{9} \mathrm{CH}_{3}(\mathrm{C} 10)$ & $2.6 \pm 0.3$ & $18-28^{\circ}$ & & $5.7 \pm 0.7$ & $62-80^{\circ}$ \\
$\equiv \mathrm{Si}-\left(\mathrm{CH}_{2}\right)_{11} \mathrm{CH}_{3}(\mathrm{C} 12)$ & $2.6 \pm 0.4$ & $18-28^{\circ}$ & & $4.4 \pm 0.4$ & $46-66^{\circ}$ \\
$\equiv \mathrm{Si}-\left(\mathrm{CH}_{2}\right)_{13} \mathrm{CH}_{3}(\mathrm{C} 14)$ & $2.5 \pm 0.2$ & $18-28^{\circ}$ & & $4.1 \pm 0.6$ & $41-49^{\circ}$ \\
$\equiv \mathrm{Si}-\left(\mathrm{CH}_{2}\right)_{17} \mathrm{CH}_{3}(\mathrm{C} 18)$ & $2.4 \pm 0.3$ & $18-28^{\circ}$ & & $3.3 \pm 0.7$ & $33-36^{\circ}$
\end{tabular}

deposition and their values range from $18^{\circ}$ to $28^{\circ}$, independent of the different alkyl chain lengths. On the other hand, the tilt angles of methyl groups for samples prepared via sputtering deposition exhibit chain-length dependence: their tilt becomes smaller as the chain length increases from C10 to C18. Such remarkable variations indicate that different structural changes are induced during the deposition of gold overlayers (by sputtering vs thermal evaporation) on identically prepared $n$-alkyl monolayers on silicon.

In the case of thermal evaporation, the solid metal melts and evaporates in the crucible mounted at the bottom of a vacuum chamber. The metal vapor consequently condenses and deposits onto the sample, inducing a certain level of damage to the organic monolayer because of the energy released from of the gradually condensing atoms. ${ }^{46}$ The observed identical, but small, tilt angle of alkyl monolayers on silicon buried underneath the gold contacts may be due to thermal expansion during the gold evaporation process, a known phenomenon. ${ }^{42,47}$ This means that irrespective of the chain length, all monolayers stand up vertically on the substrate upon metal deposition; that is, the methyl tilt angles become much smaller in comparison to the values prior to metal deposition. The thermal radiation of the boiling gold source $(>3000 \mathrm{~K})$ makes the molecules more mobile, as evidenced by the new $\mathrm{CH}_{2}$ vibration band (Scheme $1)$.

In sputtering deposition, gold atoms are "dislocated" from the source (target) as struck by the high-energy ions generated by magnetron-assisted DC plasma. The "emitted" high-energy, neutral metal atoms travel toward the sample situated on the opposite side of the chamber. Sputtering is considered to be more energetic than thermal evaporation, as the kinetic energy of ions is transferred to the ejected metal atoms. Sputtered atoms ejected from the target have a wide energy distribution, typically tens of electronvolts, which is much higher than the thermal

SCHEME 1: Hypothetical View of Monolayer-Metal Interactions during Gold Deposition via (a) Thermal Evaporation and (b) Sputtering Deposition

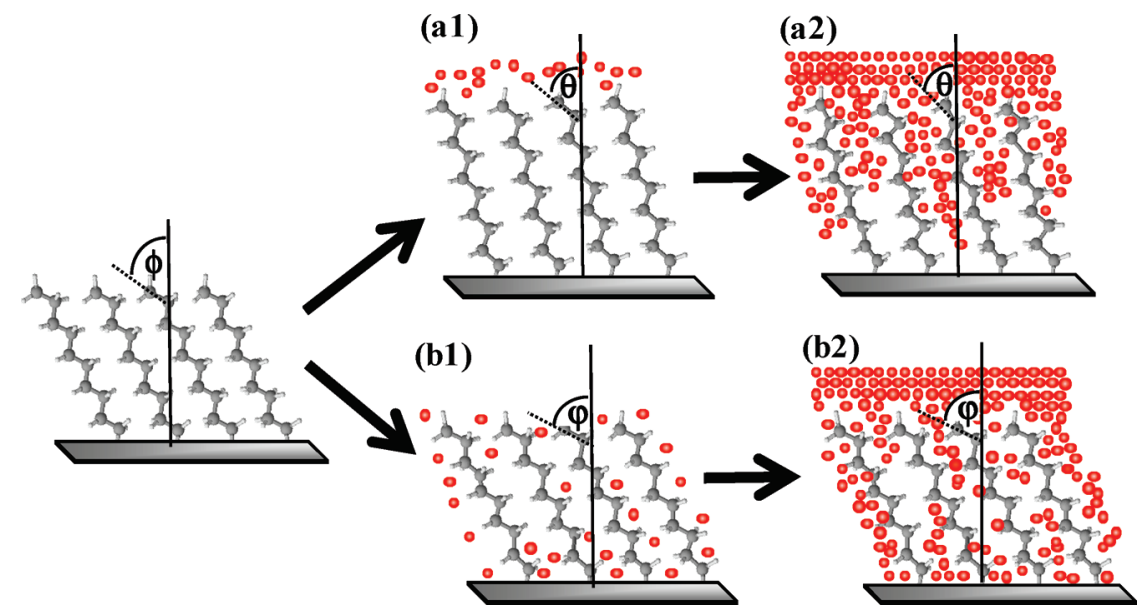



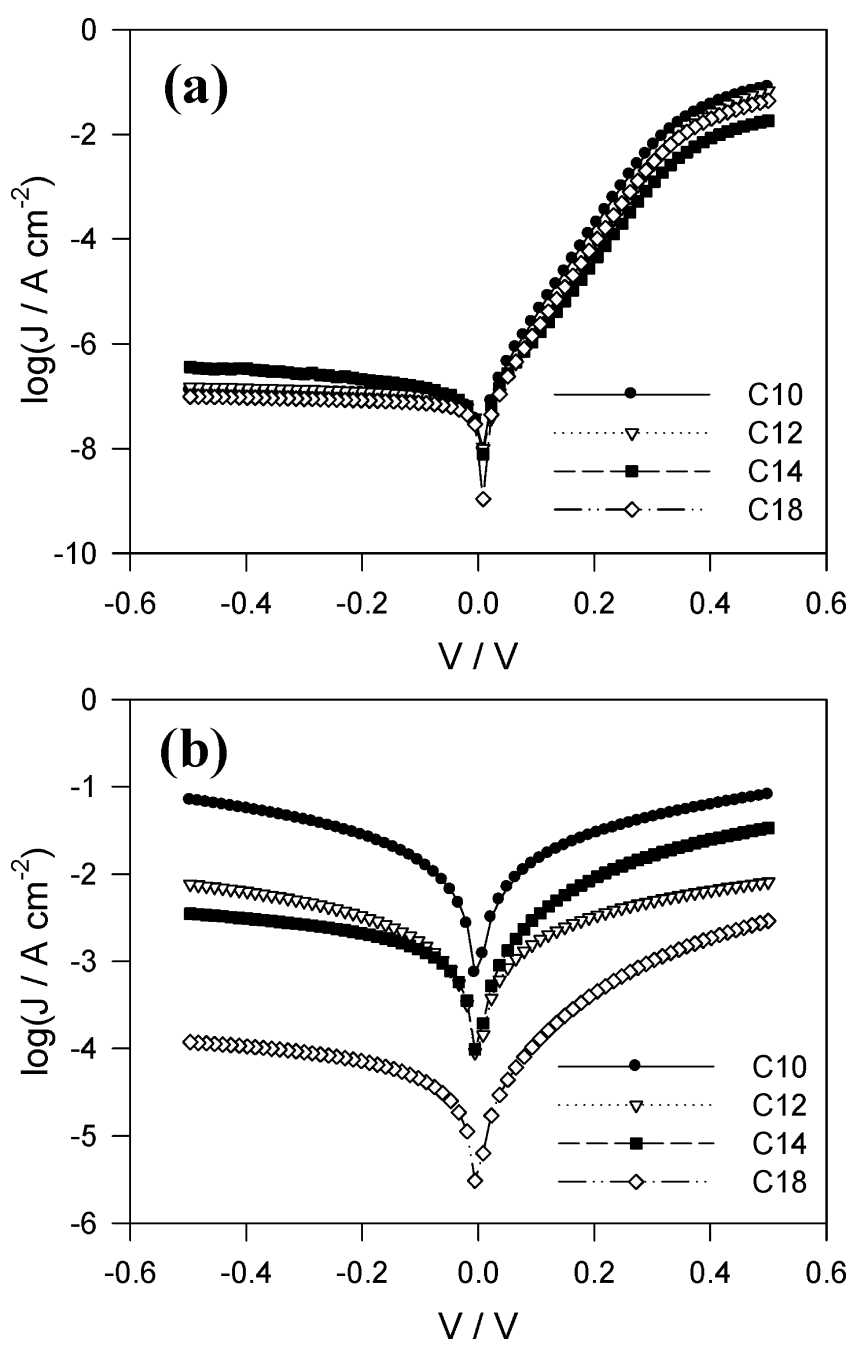

Figure 4. Current density-bias voltage $(J-V)$ plots of Au/alkyl monolayer/Si junctions, for which the alkyl chain length was systematically varied. The gold contacts were prepared by (a) thermal evaporation and (b) sputtering deposition.

energy of condensing gold atoms $(\sim 0.27 \mathrm{eV})$. Thermal expansion of the monolayer will be minimized in this case because the source is kept cold; however, the high kinetic energies allow the metal atoms to penetrate the monolayers more readily than in the thermal evaporation process (Scheme 1, b1). The different tilt angles upon sputtering gold on top of alkyl monolayers on silicon indicate that the monolayers respond to the bombardment with high-energy gold atoms differently. Although a short-chain monolayer (i.e., C10) will simply maintain its original tilt angle, the longer alkyl chains may be reoriented upon metal penetration. As the gaps between molecules are occupied slowly by metal atoms, the molecules gradually stand up. The affinity of methylene groups for gold atoms has been demonstrated previously; ${ }^{35}$ thus, longer alkyl chains will limit the mobility of gold atoms within the monolayer.

3.4. Electrical Measurements. It is evident that both deposition methods result in gold penetration into the monolayers and induce gauche defects within alkyl-chain backbones (Scheme 1). However, the structural deformation of the organic monolayers at the buried interfaces differs significantly, as suggested by the above SFG investigations. The structural differences are also reflected in the electrical properties of thus formed $\mathrm{Au} /$ alkyl monolayer/Si junctions. Figure 4 shows typical current density $(J)$ versus bias voltage $(V)$ plots. All samples prepared
TABLE 3: Solid-State Electrical Characterization of Au/Alkyl Monolayer/Si Junctions

\begin{tabular}{lcccc}
\hline \multicolumn{1}{c}{ sample } & $n$ & $\mathrm{q} \phi_{\text {eff }} / \mathrm{eV}$ & $N_{\mathrm{d}} / 10^{15} \mathrm{~cm}^{-3}$ & $V_{\mathrm{D} 0} / \mathrm{V}$ \\
\hline$\equiv \mathrm{Si}-\left(\mathrm{CH}_{2}\right)_{9} \mathrm{CH}_{3} / \mathrm{Au}(\mathrm{C} 10)$ & $1.21 \pm 0.29$ & $0.83 \pm 0.06$ & $2.27 \pm 0.41$ & $-0.51 \pm 0.03$ \\
$\equiv \mathrm{Si}-\left(\mathrm{CH}_{2}\right)_{11} \mathrm{CH}_{3} / \mathrm{Au}(\mathrm{C} 12)$ & $1.20 \pm 0.26$ & $0.87 \pm 0.05$ & $2.17 \pm 0.87$ & $-0.63 \pm 0.08$ \\
$\equiv \mathrm{Si}-\left(\mathrm{CH}_{2}\right)_{13} \mathrm{CH}_{3} / \mathrm{Au}(\mathrm{C} 14)$ & $1.31 \pm 0.21$ & $0.89 \pm 0.03$ & $1.84 \pm 0.29$ & $-0.59 \pm 0.01$ \\
$\equiv \mathrm{Si}-\left(\mathrm{CH}_{2}\right)_{17} \mathrm{CH}_{3} / \mathrm{Au}(\mathrm{C} 18)$ & $1.25 \pm 0.38$ & $0.86 \pm 0.02$ & $1.89 \pm 0.32$ & $-0.55 \pm 0.03$
\end{tabular}

via thermal deposition exhibited clear rectifying behavior (Figure 4a). The effective barrier heights and ideality factors were determined based on the thermionic emission model (see the Supporting Information) and are summarized in Table 3. The average values of $\mathrm{q} \phi_{\text {eff }}$ for the four monolayer systems are slightly different; that is, a marginal increase was observed for C10-C14, but the value dropped for C18. Such an insignificant increase may be due to the depth (extent) of gold penetration being dependent on the monolayer thickness. The drop in $\mathrm{q} \phi_{\text {eff }}$ for the C18 monolayer can be explained by its lower packing density, as indicated by the smaller water contact angle (106 \pm $2^{\circ}$ ). It is important to note that most of the $\mathrm{q} \phi_{\text {eff }}$ values were within the experimental uncertainties. We have shown previously that there is no dependence on the alkyl chain length when the monolayers are prepared via an organometallic route (i.e., identical to an $\mathrm{Au} / \mathrm{H}-\mathrm{Si}$ junction). ${ }^{48}$ In addition, the rather large uncertainties compared with those previously reported for $\mathrm{Hg}$ / alkyl monolayer/Si junctions ${ }^{49}$ indicate the uneven lateral gold penetration. This is consistent with our SFG observations; that is, there are $\mathrm{C}-\mathrm{H}$ groups that are not in direct contact with the gold atoms.

In contrast, the junctions prepared via sputtering deposition were less rectifying and the current densities clearly different when the chain length was varied (Figure $4 b$ ). The semilog $J-V$ curve for $\mathrm{C} 10$ is symmetric, which means that such a junction is essentially an Ohmic contact. As the chain length increases, the rectifying behavior becomes more prominent (i.e., the current at forward bias becomes much higher than that at the reversed bias). In principle, a direct $\mathrm{Au} / \mathrm{Si}$ junction should be rectifying, as shown in our previous report. ${ }^{48}$ The Ohmic behavior of the $\mathrm{Au} / \mathrm{H}-\mathrm{Si}$ contact has been previously observed by scanning tunneling microscopy (STM) and attributed to the induced surface states in the band gap region as a result of direct bonding between gold and silicon. ${ }^{50}$ The Ohmic behavior of sputtered samples indicates strong interactions between gold atoms and the silicon substrates; that is, high-energy gold atoms penetrate the monolayers and attack the silicon surface, possibly with shallow penetration into the silicon bulk. It is interesting to note that, although the penetration occurs to a greater extent, the alkyl chains stay intact and relatively well-oriented. Gold atoms are not displacing the surface silicon atoms since such damage would cause a dislocation of the monolayer. As the chain length increases, the degree of gold penetration seems to decrease; however, it is more significant than in thermal evaporation where all junctions showed clear rectifying behavior. This result supports the SFG data, suggesting that metal penetration occurs at a slower rate for the longer alkyl chains (reflected by the difference in the tilting angles of the buried monolayers). The loss of a clear $\mathrm{Au}-($ molecule $)-\mathrm{Si}$ interface was also supported by the fact that linear Mott-Schottky $(\mathrm{M}-\mathrm{S})$ curves were not obtained for sputtered samples, whereas for samples prepared via thermal evaporation, the $\mathrm{M}-\mathrm{S}$ plots were linear over a large potential range (Figure 5), an indication of intact silicon substrates. In particular, the doping density $\left(N_{\mathrm{d}}\right)$ and built-in potential at zero bias $\left(V_{\mathrm{D} 0}\right)$, estimated from $\mathrm{M}-\mathrm{S}$ plots (Table 3 ), are in good agreement with previously reported data. ${ }^{48,51}$ 


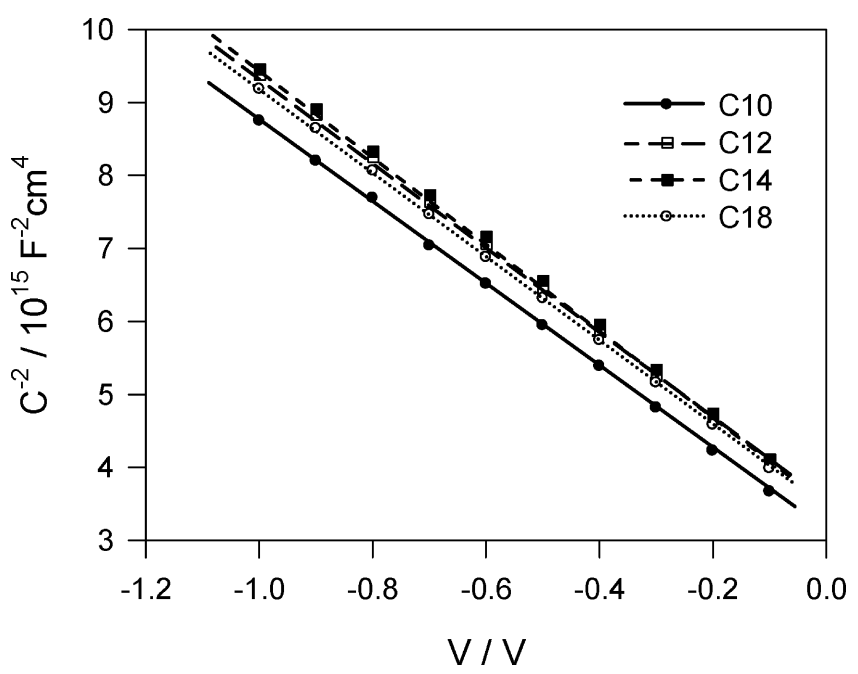

Figure 5. Mott-Schottky $(\mathrm{M}-\mathrm{S})$ plots of Au/alkyl monolayer/Si junctions; the gold contacts were prepared via thermal evaporation.

\section{Conclusion}

We have shown that SFG spectroscopy is a powerful tool to probe the molecular order of organic monolayers at buried metal/ semiconductor interfaces. Gold penetration induces gauche defects to the alkyl monolayers prepared on silicon, but the monolayers surprisingly maintain relatively ordered configurations. The method of gold deposition (thermal evaporation vs sputtering) affects the monolayer structure at the buried interface. The average tilt angle of methyl groups was similar $\left(\sim 28^{\circ}\right)$ for all samples prepared via thermal evaporation, whereas for the sputtered samples, chain-length dependence was evident. In both cases, the monolayers seem to "stand up" upon gold deposition; for the thermal evaporation, this can be explained by the "heating effect" of the radiation source. The sputtering process induces more permanent damage and disordering to the alkyl monolayers; thus formed junctions even lose the rectifying property as a result of strong interactions between gold atoms and the silicon substrate.

Acknowledgment. We wish to thank the Natural Science and Engineering Research Council (NSERC) of Canada for financial support. This work was also supported by the Global COE Program (Project No. B01: Catalysis as the Basis for Innovation in Materials Science) for the Promotion of Novel Interdisciplinary Fields Based on Nanotechnology and Materials and by Grants-in-Aid for Young Scientists (B) (No. 19750054) and for Scientific Research (A) (No. 18205016) from the Ministry of Education, Culture, Sports, Science, and Technology (MEXT), Japan. H.A. is grateful for the experimental help and valuable suggestions from Mikio Ito and Tsubasa Okada. H.-Z.Y. thanks the Japan Society for the Promotion of Science (JSPS) for supporting his sabbatical stay in Sapporo through an Invitation Fellowship and Dr. Eberhard Kiehlmann for editing the manuscript.

Supporting Information Available: Experimental results of ATR-FTIR and solid-state electrical measurements. This material is available free of charge via the Internet at http:// pubs.acs.org.

\section{References and Notes} $59,1597$.
(2) Harris, A. L.; Chidsey, C. E. D.; Levinos, N. J.; Loiacono, D. N. Chem. Phys. Lett. 1987, 141, 350.

(3) Hunt, J. H.; Guyotsionnest, P.; Shen, Y. R. Chem. Phys. Lett. 1987, $133,189$.

(4) Zhu, X. D.; Suhr, H.; Shen, Y. R. Phys. Rev. B 1987, 35, 3047.

(5) Richmond, G. L. Chem. Rev. 2002, 102, 2693.

(6) Shen, Y. R. Nature 1989, 337, 519.

(7) Chen, C. Y.; Loch, C. L.; Wang, J.; Chen, Z. J. Phys. Chem. B 2003, 107, 10440 .

(8) Guyotsionnest, P.; Superfine, R.; Hunt, J. H.; Shen, Y. R. Chem. Phys. Lett. 1988, 144, 1. 493.

(9) Hines, M. A.; Todd, J. A.; Guyotsionnest, P. Langmuir 1995, 11,

(10) Ishibashi, T.; Ara, M.; Tada, H.; Onishi, H. Chem. Phys. Lett. 2003, $367,376$.

(11) Liu, Y.; Wolf, L. K.; Messmer, M. C. Langmuir 2001, 17, 4329.

(12) Nihonyanagi, S.; Miyamoto, D.; Idojiri, S.; Uosaki, K. J. Am. Chem. Soc. 2004, 126, 7034.

(13) Nishi, N.; Hobara, D.; Yamamoto, M.; Kakiuchi, T. J. Chem. Phys. 2003, 118, 1904.

(14) Ye, S.; Nihonyanagi, S.; Uosaki, K. Phys. Chem. Chem. Phys. 2001, 3,3463 .

(15) Ye, S.; Saito, T.; Nihonyanagi, S.; Uosaki, K.; Miranda, P. B.; Kim, D.; Shen, Y. R. Surf. Sci. 2001, 476, 121.

(16) Yeganeh, M. S.; Dougal, S. M.; Polizzotti, R. S.; Rabinowitz, P. Phys. Rev. Lett. 1995, 74, 1811.

(17) Flood, A. H.; Stoddart, J. F.; Steuerman, D. W.; Heath, J. R. Science 2004, 306, 2055.

(18) de Boer, B.; Frank, M. M.; Chabal, Y. J.; Jiang, W. R.; Garfunkel, E.; Bao, Z. Langmuir 2004, 20, 1539.

(19) Walker, A. V.; Tighe, T. B.; Cabarcos, O. M.; Reinard, M. D.; Haynie, B. C.; Uppili, S.; Winograd, N.; Allara, D. L. J. Am. Chem. Soc. 2004, 126, 3954

(20) Jun, Y. S.; Zhu, X. Y. J. Am. Chem. Soc. 2004, 126, 13224.

(21) Richter, C. A.; Hacker, C. A.; Richter, L. J. J. Phys. Chem. B 2005, $109,21836$.

(22) Hacker, C. A.; Richter, C. A.; Gergel-Hackett, N.; Richter, L. J. J. Phys. Chem. C 2007, 111, 9384.

(23) Scott, A.; Hacker, C. A.; Janes, D. B. J. Phys. Chem. C 2008, 112, 14021 .

(24) Linford, M. R.; Fenter, P.; Eisenberger, P. M.; Chidsey, C. E. D. J. Am. Chem. Soc. 1995, 117, 3145.

(25) Asanuma, H.; Noguchi, H.; Uosaki, K.; Yu, H.-Z. J. Phys. Chem. B 2006, 110, 4892.

(26) Strunskus, T.; Grunze, M.; Kochendoerfer, G.; Woll, C. Langmuir 1996, 12, 2712.

(27) Shen, Y. R. The Principles of Nonlinear Optics; John Wiley \& Sons: New York, 1984.

(28) Faber, E. J.; de Smet, L. C. P. M.; Olthuis, W.; Zuilof, H.; Sudholter, E. J. R.; Bergveld, P.; van den Berg, A. ChemPhysChem 2005, 6, 2153. (29) Ishizaki, T.; Saito, N.; SunHyung, L.; Ishida, K.; Takai, O. Langmuir 2006, 22, 9962.

(30) Sieval, A. B.; Vleeming, V.; Zuilhof, H.; Sudholter, E. J. R. Langmuir 1999, 15, 8288.

(31) Harris, A. L.; Chidsey, C. E. D.; Levinos, N. J.; Loiacono, D. N. J. Electrochem. Soc. 1988, 135, C386.

(32) Tanaka, Y.; Lin, S.; Aono, M.; Suzuki, T. Appl. Phys. B: Laser Opt. 1999, 68, 713 .

(33) Messmer, M. C.; Conboy, J. C.; Richmond, G. L. J. Am. Chem. Soc. $1995,117,8039$.

(34) Yamamoto, M.; Sakurai, Y.; Hosoi, Y.; Ishii, H.; Ito, E.; Kajikawa, K.; Ouchi, Y.; Seki, K. Surf. Sci. 1999, 428, 388.

(35) Backus, E. H. G.; Bonn, M. Chem. Phys. Lett. 2005, 412, 152.

(36) Zhu, Z.; Daniel, T. A.; Maitani, M.; Cabarcos, O. M.; Allara, D. L.; Winograd, N. J. Am. Chem. Soc. 2006, 128, 13710.

(37) Haick, H.; Niitsoo, O.; Ghabboun, J.; Cahen, D. J. Phys. Chem. C 2007, 111, 2318.

(38) Nakamura, T.; Miyamae, T.; Yoshimura, D.; Kobayashi, N.; Nozoye, H.; Matsumoto, M. Langmuir 2005, 21, 5026.

(39) Noguchi, H.; Ito, M.; Uosaki, K. Chem. Lett. 2005, 34, 950.

(40) Braun, R.; Casson, B. D.; Bain, C. D.; van der Ham, W. M.; Vrehen, Q. H. F.; Ellel, E. R.; Briggs, A. M.; Davies, P. B. J. Chem. Phys. 1999, 110,4634

(41) Watanabe, N.; Yamamoto, H.; Wada, A.; Domen, K.; Hirose, C. Spectrochim. Acta, Part A 1994, 50A, 1529.

(42) Zhang, L. Z.; Wesley, K.; Jiang, S. Y. Langmuir 2001, 17, 6275.

(43) Gautam, K. S.; Schwab, A. D.; Dhinojwala, A.; Zhang, D.; Dougal,

S. M.; Yeganeh, M. S. Phys. Rev. Lett. 2000, 85, 3854.

(44) Wang, J.; Chen, C. Y.; Buck, S. M.; Chen, Z. J. Phys. Chem. B 2001, 105, 12118 .

(45) Ye, S.; Morita, S.; Li, G. F.; Noda, H.; Tanaka, M.; Uosaki, K.; Osawa, M. Macromolecules 2003, 36, 5694. 
(46) Haick, H.; Cahen, D. Prog. Surf. Sci. 2008, 83, 217-261.

(47) Salomon, A.; Shpaisman, H.; Seitz, O.; Boecking, T.; Cahen, D. J. Phys. Chem. C 2008, 112, 4169.

(48) Kuikka, M. A.; Li, W. J.; Kavanagh, K. L.; Yu, H.-Z. J. Phys. Chem. C 2008, 112, 9081.

(49) Liu, Y.-J.; Yu, H.-Z. ChemPhysChem 2002, 3, 799.
(50) Gheber, L. A.; Hershfinkel, M.; Gorodetsky, G.; Volterra, V. Thin Solid Films 1998, 320, 228.

(51) Asanuma, H.; Bishop, E. M.; Yu, H.-Z. Electrochim. Acta 2007, 52, 2913.

JP9062675 